\title{
A propos de fin de vie
}

\section{Jean Martin}

Dr méd., membre de la rédaction

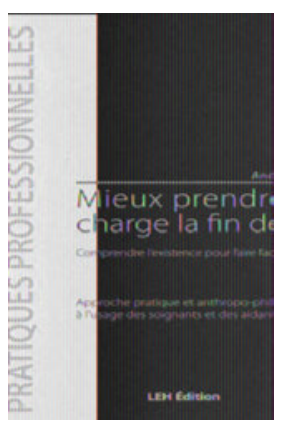

André Marro

Mieux prendre en charge la fin de vie

Comprendre l'existence pour faire face à la mort Bordeaux: LEH Edition; 2017.

160 pages. $37 \mathrm{CHF}$.

ISBN 9782848747385

Dans la préface du psychiatre Patrick Lemoine: «Cet ouvrage est complètement original et apporte un éclairage nouveau à la difficile question de la position existentielle des aidants. Ni métier, ni bénévolat, leur mission est à haut risque, leur désarroi n'est pas une fiction [...] Le conseil de l'auteur est simple et lumineux: chercher à adopter une juste et salutaire distance professionnelle afin de ne pas s'épuiser, pour être des facilitateurs de vie et de soins. Cette manière d'être ressemble à une mystique existentielle et demande un 'chavirement' dans sa manière d'être.»

André Marro, enseignant universitaire à Nice, est paléo-anthropologue et formateur, surtout dans le domaine médico-social, en sciences et techniques de communication (tout en ayant «un goût certain pour le silence»). Deux chapitres à son livre. Le premier est «Posture de l'aidant face au mystère de la souffrance et de la fin de vie». Dans ses sections successives, on traite de divers aspects de la souffrance, y compris de sa métaphysique, des textes français récents sur la fin de vie (loi Leonetti, rapport Sicard de 2012), de distance professionnelle et sociale (proxémie), des mécanismes de défense que les soignants tendent à mettre en place dans leur relation avec les malades. Puis sont abordées diverses techniques de "pleine conscience» - méditation, marche consciente, zen, yoga -, les états de conscience modifiés et l'énergie vitale. Enfin une discussion de "Réussir sa relation, une expérience existentielle à l'aide de l'autre».

Le chapitre 2, "Réflexions anthropo-philosophiques», est introduit par «Si la vie a du sens, elle se devine au cœur de l'étonnement d'exister. Le seul choix possible est de tenter de comprendre la meilleure manière d'exister [...] Tout dans l'univers, de l'infiniment grand à l'infiniment petit, est oscillation. D'un côté, nous avons un goût certain pour l'imagination, de l'autre nous manions la froide pensée et la réflexion ration- nelle.» Contenu historique et philosophique, avec trois parties (chacune de 15-25 pages): «La voie théologique», discutant les croyances religieuses depuis la préhistoire et leurs mécanismes; "La voie matérialiste» ou positiviste; «La voie médiane ou de spiritualité laïque» - cette dernière citant notamment Spinoza, Nietzsche, Bergson ainsi que les philosophes grecs. Noter que ce second chapitre, qui met l'accent sur notre nature, existence et essence, est sans références directes aux soins prodigués aux malades. On y cherche, dit l'auteur, «à amenuiser la dimension inconsolable et inquiète de notre espèce en errance face à son irrévocable condition mortelle».

Quelques extraits. «Notre nature est constituée de particules élémentaires en interaction avec le vide. A cet égard, elle échappe au temps, nous sommes en quelque sorte structurellement éternels.» "Accompagner la personne vers son éternité peut être relativement simple si nous savons expérimenter notre propre éternité.»

«La plupart des aidants disent qu'il y a quelque chose d'émouvant et même de ressourçant à accompagner la vulnérabilité d'une personne en souffrance [...] Cette rencontre permet à l'aidant de se libérer des conditionnements de la culture et du social.» A propos des risques de burn-out des soignants: "S'il y a quelque chose à dénoncer, ce serait le vide philosophique et existentiel de nos sociétés dites modernes face à leur devoir de formation et réflexion sur les grands mystères tels que la démence ou la mort.»

Intéressé à la problématique, j'ai pensé en apprenant la sortie de ce livre qu'il apportait surtout des contributions sur la pratique au lit du patient en fin de vie. Il y a un peu de cela mais l'essentiel est fait de réflexions sur la condition, non pas de malade, mais de soignant/aidant; sur sa posture et la lutte contre l'épuisement. Les soins aux malades n'apparaissent guère et on comprend l'auteur dans son préambule disant: «Cet ouvrage peut être considéré comme un manuel pour les soignants comme pour tous ceux qui cherchent leur véritable liberté.» Il eût été judicieux d'indiquer/avertir qu'est peu traitée l'activité quotidienne au côté de personnes vivant l'ultime étape de leur vie. En fait, le sous-titre actuel de l'ouvrage aurait pu avantageusement être choisi comme son titre. 